\title{
Triple-Wavelength Fiber Ring Laser Based on a Hybrid Gain Medium Actively Mode-locked at $10 \mathrm{GHz}$
}

\author{
C. O’Riordan*, M.J. Connelly*, P.M. Anandarajah**, R. Maher**, L.P. Barry** \\ * Optical Communications Research Group, Department of Electronic and Computer Engineering, University of \\ Limerick, Limerick, Ireland \\ ** Radio and Optical Communications Group, Research Institute for Networks and Communications Engineering, \\ School of Electronic Engineering, Dublin City University, Dublin 9, Ireland \\ colm.oriordan@ul.ie, michael.connelly@ul.ie, anandara@eeng.dcu.ie, robert.maher@eeng.dcu.ie, liam.barry@dcu.ie
}

\begin{abstract}
A fiber ring laser based on a hybrid gain medium that produces three simultaneously mode-locked wavelength channels is presented. The Lithium Niobate based modulator used to actively mode-lock the laser cavity at $\mathbf{1 0}$ $\mathrm{GHz}$ is birefringence compensated to reduce its polarization sensitivity. A Lyot filter defines the lasers multiwavelength spectrum which has a wavelength spacing of $1 \mathrm{~nm}$. The polarization sensitive nature of the laser cavity and its affect on the performance of the laser is discussed.
\end{abstract}

\section{INTRODUCTION}

Multiwavelength fiber ring lasers (MWFRL) have attracted a significant amount of research interest due to the cost saving benefit of using a single device to generate numerous carrier wavelengths in wavelength division multiplexed (WDM) transmission systems. By actively mode-locking the MWFRL cavity a high repetition rate multiwavelength short optical pulse source may be realized [1]. This multiwavelength pulse source can be combined with time division multiplexing (TDM) techniques to increase the transmission capacity of optical fiber thereby highlighting applications in high speed optical communications systems. Such sources also have applications in optical gas sensing, optical instrumentation and optical signal processing.

We present a MWFRL using a hybrid gain medium consisting of an erbium-doped fiber amplifier (EDFA) and a semiconductor optical amplifier (SOA). The inhomogeneous gain medium of the SOA serves to suppress the homogenous line broadening of the EDFA allowing for the generation of numerous closely spaced wavelength channels [2]. This is a far more practical solution than cooling the EDFA in liquid nitrogen as proposed in [3]. An additional benefit is that the supermode noise that is produced when the laser is harmonically mode-locked is reduced due to the fast gain saturation profile of the SOA [4], [5]. The incorporation of a SOA in a harmonically mode-locked MWFRL also provides the facility to actively modulate the SOA. A Lyot filter is used to force multiwavelength operation of the laser [6]. The length of polarization maintaining fiber used in the Lyot filter determines the wavelength spacing. The MWFRL is harmonically actively mode-locked using an intra-cavity $\mathrm{LiNbO}_{3}$ based Mach-Zehnder intensity modulator (MZM). The MZM is birefringence

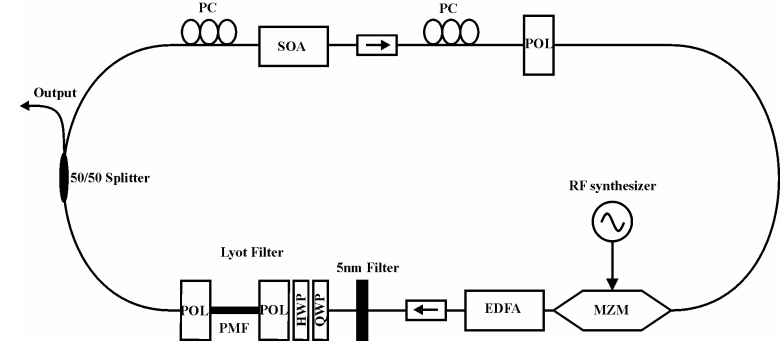

Figure 1. Layout of the MWFRL. POL: polarizer; HWP: half wave plate; QWP: quarter wave plate; PC: polarization controller; PMF: polarization maintaining fiber; MZM: Mach-Zehnder modulator; SOA: semiconductor optical amplifier; EDFA: erbium-doped fiber amplifier.

compensated to account for its polarization sensitivity [6] The laser cavity exhibits significant polarization sensitivity due to the use of the polarization dependent Lyot filter and the MZM. This polarization sensitivity is explained in more detail and its affect on the performance of the MWFRL is discussed.

\section{EXPERIMENTAL SETUP}

The configuration of the MWFRL is shown in Fig. 1. The laser cavity is constructed from standard single modefiber (SMF). The SOA (Kamelian OPA-20-N-C-FA) is a commercially available fiber pigtailed device that has a typical small signal gain of $22 \mathrm{~dB}$, a saturation output power of $13 \mathrm{dBm}$ and a typical polarization dependent gain of $0.19 \mathrm{~dB}$ at $1550 \mathrm{~nm}$. The EDFA has a saturated output power of $12 \mathrm{dBm}$ and a small signal gain of approximately $30 \mathrm{~dB}$ at $1550 \mathrm{~nm}$. $\mathrm{A} \mathrm{LiNb0}_{3}$ based MZM is used to actively mode-lock the MWFRL cavity. MZM birefringence compensation is realised by inserting a polarizer directly before the MZM [6]. In order to maximize the output power from the laser the lossiest element in the ring, the MZM, is positioned before the EDFA. The polarization controllers (PC) control the lasing polarization. A Lyot filter is used to generate the multiwavelength spectrum. A wavelength channel spacing of $1 \mathrm{~nm}$ is obtained when $4 \mathrm{~m}$ of PMF is inserted in the Lyot filter. Quarter and half wave plates (QWP, HWP) are inserted directly before the first polarizer of the Lyot filter to ensure favorable alignment of the laser light state of polarization (SOP) through the Lyot filter. Isolators are utilized to ensure unidirectional operation of the laser. 


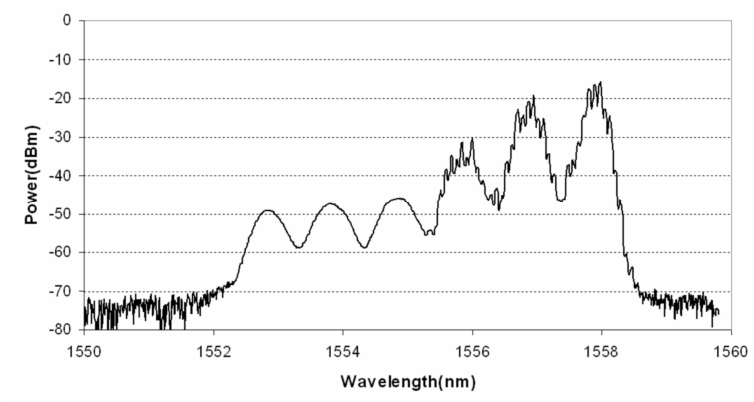

(a)
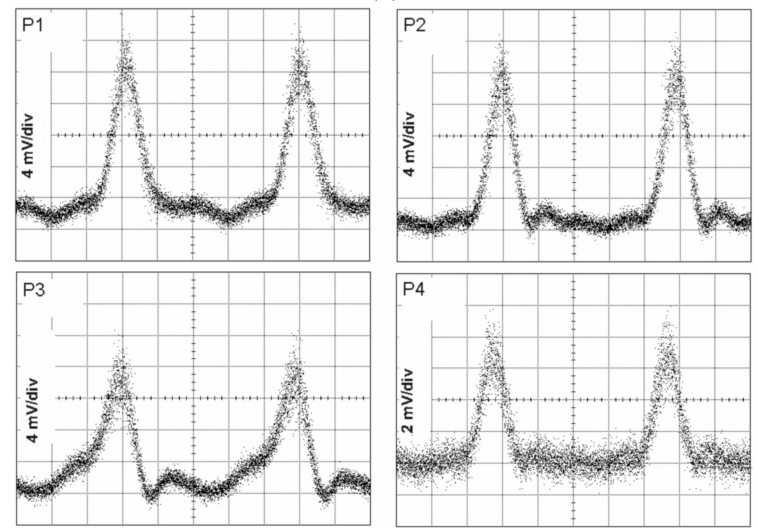

(b)

Figure 2. (a) Triple-wavelength mode-locked spectrum. (b) P1: composite pulse train; P2, P3, P4: pulse trains of the filtered wavelengths. Time base is $20 \mathrm{ps} / \mathrm{div}$.

A $5 \mathrm{~nm}$ filter is used to limit the number of wavelengths circulating in the laser cavity. A $3 \mathrm{~dB}$ splitter is tap off a portion of the laser light for characterization using an optical spectrum analyzer and a digital communications analyzer.

\section{RESULTS AND DISCUSSION}

The MWFRL was actively mode-locked at approximately $10 \mathrm{GHz}$ by driving the MZM at a harmonic of the laser cavity fundamental frequency which was 3.15 MHz. Through optimization of laser light SOP three wavelength channels are simultaneously mode-locked. The MWFRL spectrum can be seen in Fig. 2a. The modulation profile at $10 \mathrm{GHz}$ can be clearly seen on three of the wavelength channels. To confirm the presence of pulses at each wavelength a tunable filter is used to discriminate each of the mode-locked wavelengths. The composite pulse train as well as the pulse train at each mode-locked wavelength is shown in Fig. 2b. As expected the individual pulse trains are of the same repetition rate and are synchronous with the composite pulse train. The pulse width achieved was approximately $15 \mathrm{ps}$. The wavelength spacing is approximately $1 \mathrm{~nm}$. ITU specified wavelength spacing's may also be obtained as indicated by our previous work [6], [7]. However, due to the relatively poor extinction of the tunable filter, broader wavelength spacing is required to ensure adequate rejection of neighboring wavelength channels.

It is extremely difficult to generate more than 3 modelocked wavelength channels. The reason is that when many oscillating wavelengths enter the SOA

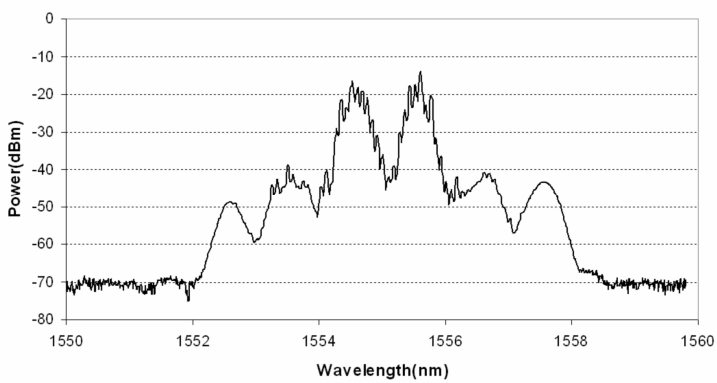

(a)

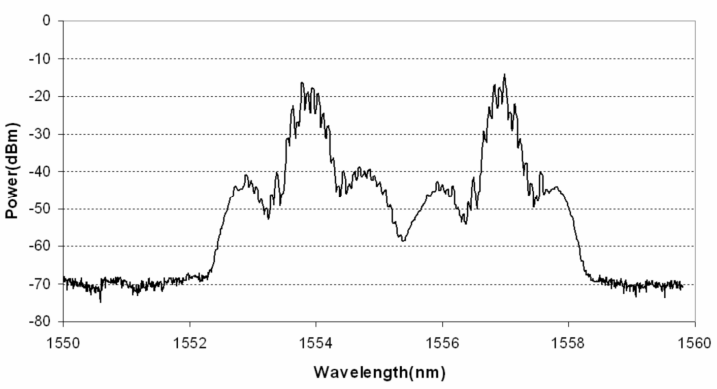

(b)

Figure 3. (a), (b) Dual-wavelength mode-locked spectra highlighting the result of adjusting the laser cavity SOP

simultaneously a substantial number of carriers are consumed and this leads to competition between the wavelengths for gain. Only those wavelengths that experience enough gain to over come the cavity losses will have sufficient energy to mode-lock. These wavelengths are selected by adjusting the laser cavity SOP thereby transferring the energy needed for mode-locking between the various wavelengths [8]. Fig. 3a and Fig. 3b highlights the affect of adjusting the laser cavity SOP on the mode-locked MWFRL spectrum. In order to limit the number of wavelength channels generated, a $5 \mathrm{~nm}$ filter is placed in the laser cavity. This helps to maximize the number of wavelengths experiencing enough gain to sustain mode-locked operation as the overall number of wavelengths competing for gain is reduced. As different wavelengths may have different SOP, different gains may be experienced by each of the wavelengths. However as the polarization dependent gain of the SOA used in this MWFRL is only $0.19 \mathrm{~dB}$ this should not be a significant issue.

The $\mathrm{LiNb}_{3}$ based MZM is a polarization sensitive device. A simple technique was presented in [6] whereby a polarizer is inserted before the MZM whose polarization axis is aligned with one of the birefringence axes of the MZM. This effectively matches the laser light SOP with one of the birefringence axes of the MZM. The result is a MZM with reduced polarization sensitivity. A Lyot filter is a polarization dependent interference filter which generates a periodic wavelength dependent transmission response. When used in conjunction with a $\mathrm{LiNbO}_{3}$ based MZM the result is a highly polarization sensitive laser cavity. It was observed that it is extremely difficult to optimize the laser cavity for triple and dual-wavelength mode-locking. The Lyot filter transmission response is optimized by adjusting the QWP, HWP and polarizer's of the Lyot filter assembly. Once the cavity is mode-locked 
the laser cavity SOP is adjusted in order to optimize the MWFRL spectrum. This involves adjusting the PC's before the SOA the MZM. This adjustment affects the favorable alignment of the laser light SOP through the Lyot filter. The Lyot filter transmission response is no longer optimized and has to be readjusted via the QWP and HWP. This readjustment in turn affects the laser cavity SOP and the optimization process starts again. Such a scenario is undesirable and does not lend itself to easy optimization of the mode-locked MWFRL spectrum while compromising the stability of the laser. Furthermore, as the laser cavity is constructed from SMF the laser cavity SOP is susceptible to environmental perturbations such as changing ambient temperatures and physical disturbances to the fiber itself. The stability of the MWFRL is further compromised as a result.

By using a polarization independent filter, such as a Fabry-Perot filter [1], to force multiwavelength operation of the laser one could expect a significant reduction in the polarization sensitivity of the MWFRL cavity. The MZM may also be replaced with a polarization insensitive modulator such as an electroabsorption modulator (EAM) or removed entirely with the SOA being used as a modulator. This may be realized through injection of an external pulse source into the laser cavity whose repetition rate is a harmonic of the lasers fundamental frequency [9], [10]. The external pulse source serves to periodically modulate the SOA carrier density thus enabling modelocked operation of the MWFRL.

\section{CONCLUSION}

In this paper a MWFRL based on a hybrid gain medium, consisting of an SOA and EDFA, has been presented. Three wavelengths channels are simultaneously mode-locked at $10 \mathrm{GHz}$ with the laser spectrum filtered to verify the existence of pulses at each wavelength. The pulse width achieved was 15 ps. The limitation on the number of mode-locked wavelengths produced may be explained by the gain competition between the oscillating wavelengths entering the SOA. The selection of the modelocked wavelengths is heavily influenced by the laser cavity SOP. Using a polarization dependent multiwavelength filter, in the form of a Lyot filter, with a MZM results is a highly polarization sensitive laser cavity. This does not lend itself to easy optimization of the MWFRL mode-locked spectrum and compromises the stability of the laser system. Using a polarization independent multiwavelength filter, such as a Fabry-Perot filter, and replacing the MZM with an EAM or actively modulating the SOA should lead to a significant reduction in the polarization sensitivity of the MWFRL.

\section{ACKNOWLEDGMENT}

The authors wish to thank the Irish Research Council for Science, Engineering and Technology (IRCSET) and Science Foundation Ireland investigator grant 02/IN1/I42 for financial support.

\section{REFERENCES}

[1] K. Vlachos, C. Bintjas, N. Pleros, H. Avramopoulos, "Ultrafast semiconductor-based fiber laser sources", IEEE Journal of Selected Topics in Quantum Electronics, vol. 10, pp. 147-154, Jan/Feb. 2004

[2] D. N. Wang, F. W. Tong, X. Fang, W. Jin, P. K. A. Wai, J. M. Gong, "Multiwavelength erbium-doped fiber ring laser source with a hybrid gain medium", Opt. Commun., vol. 228, pp. 295301, Sept. 2003.

[3] R. Hayashi, S. Yamashita, T. Saida, "16 wavelength $10 \mathrm{GHz}$ actively mode-locked fiber ring laser with demultiplexed outputs anchored on the ITU-T grid", IEEE Photonics Technol. Lett., vol. 15, pp 1692-1694, 2003.

[4] J. Yao, J. Yao, Z. Deng, "Multiwavelength actively mode-locked fibre ring laser with suppressed homogeneous line broadening and reduced supermode noise", Optics Express, vol. 12, pp. 45294534, 2004.

[5] I. Evans, C. O’Riordan, M. J. Connelly, L. P. Barry, A. M. Clarke, P. M. Anandarajah, "Investigation of noise suppression, pulse intensity and chirp of an actively mode-locked semiconductor fiber ring laser", Opt. Commun., vol. 280, pp. 142-146, 2007.

[6] C. O'Riordan, M. J. Connelly, I. Evans, P. M. Anandarajah, R. Maher, L. P. Barry, "Actively mode-locked multiwavelength fibre ring laser incorporating a Lyot filter, hybrid gain medium and birefringence compensated $\mathrm{LiNb}_{3}$ modulator", in Proc International Conference on Transparent Optical Networks, vol. 4, pp. 248-251, Jul 2007.

[7] I. Evans, M. J. Connelly, "Multiwavelength mode-locked fibre ring laser with a Lyot filter and a hybrid gain medium", in Proc. Optical Amplifiers and their Applications, Budapest, Hungary, Paper WD6, 2005.

[8] W. Zhang, J. Sun, J. Wang, L. Liu, "Multiwavelength modelocked fiber-ring laser based on reflective semiconductor optical amplifiers", IEEE Photonics Technol. Lett., vol. 19, pp. 14181420, Oct. 2007.

[9] K. Vlachos, K. Zoiros, T. Houbavlis, H. Avramopoulos, "10 simultaneously mode-locked and synchronized channels at 30 $\mathrm{GHz}$ from fiber ring laser", in Proc Optical Fiber Commun. Conf., vol. 2, pp. 344-346, Mar 2000.

[10] J. He, K.T. Chan, "All-optical actively modelocked fiber ring laser based on cross-gain modulation in SOA", Electron. Lett., vol. 38 , pp. 1504-1505, Nov. 2002. 